Revue de droit comparé du travail et de la sécurité sociale

2| 2018

Harcèlement moral au travail en jurisprudence

comparée

\title{
La lutte judiciaire contre le harcèlement au travail en Espagne : entre avancées et résistances
}

\section{Cristóbal Molina Navarrete}

\section{CpenEdition}

\section{Journals}

Édition électronique

URL : https://journals.openedition.org/rdctss/1950

DOI : $10.4000 /$ rdctss. 1950

ISSN : 2262-9815

Éditeur

Centre de droit comparé du travail et de la sécurité sociale

Édition imprimée

Date de publication : 1 juin 2018

Pagination : $70-77$

ISSN : 2117-4350

Référence électronique

Cristóbal Molina Navarrete, « La lutte judiciaire contre le harcèlement au travail en Espagne : entre avancées et résistances », Revue de droit comparé du travail et de la sécurité sociale [En ligne], 2 | 2018, mis en ligne le 01 novembre 2021, consulté le 13 novembre 2021. URL : http:// journals.openedition.org/rdctss/1950 ; DOI : https://doi.org/10.4000/rdctss.1950

\section{(c) $(7) \ominus$}

Revue de droit comparé du travail et de la sécurité sociale est mise à disposition selon les termes de la Licence Creative Commons Attribution - Pas d'Utilisation Commerciale - Pas de Modification 4.0 International. 


\section{LA LUTTE JUDICIAIRE \\ CONTRE LE HARCÈLEMENT AU TRAVAIL \\ EN ESPAGNE : ENTRE AVANCÉES ET RÉSISTANCES}
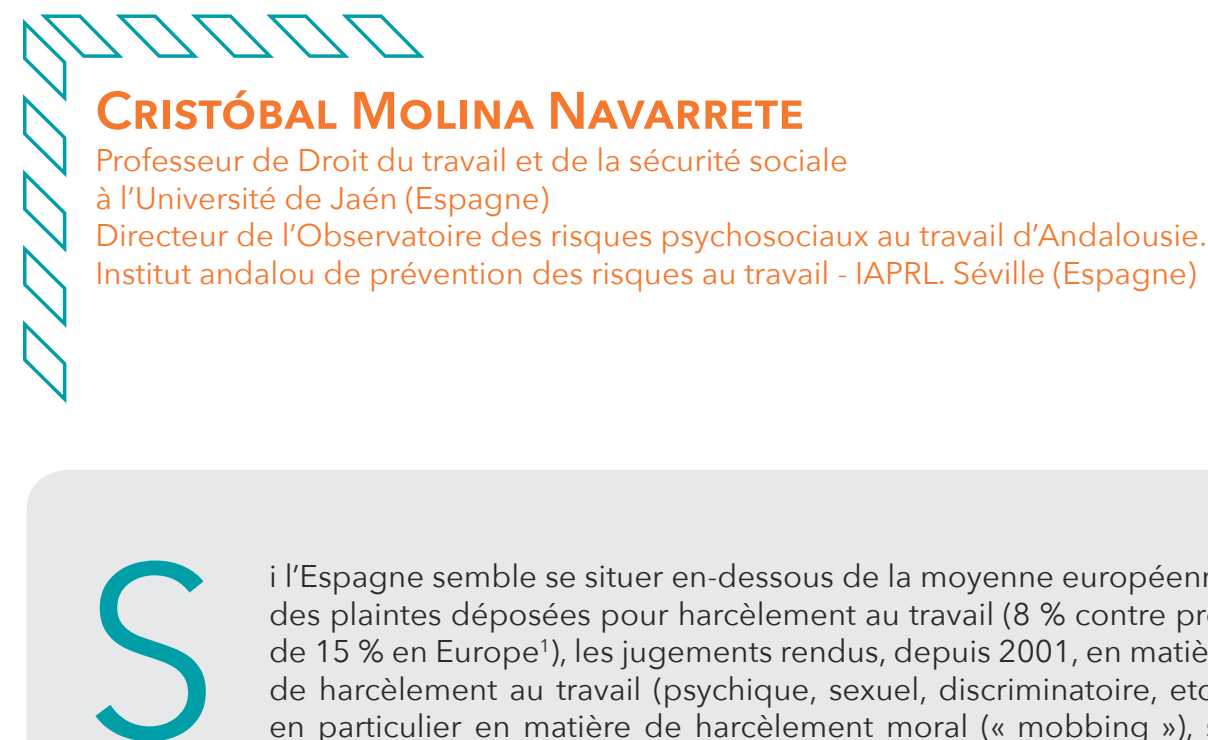

i l'Espagne semble se situer en-dessous de la moyenne européenne des plaintes déposées pour harcèlement au travail ( $8 \%$ contre près de $15 \%$ en Europe ${ }^{1}$ ), les jugements rendus, depuis 2001, en matière de harcèlement au travail (psychique, sexuel, discriminatoire, etc.), en particulier en matière de harcèlement moral (" mobbing "), se comptent par milliers. En conséquence, le système juridique espagnol, qui ne dispose pas d'une législation spécifique contre le harcèlement moral au travail, sauf dans le cadre pénal (article 173 CP), bénéficie d'une expérience importante par rapport à de tels comportements. Ce droit espagnol anti-harcèlement au travail d'origine prétorienne a rarement fait défaut, d'où l'absence persistante de législation sociale spécifique à cet égard.

Cependant, si l'abondance des décisions judiciaires relatives au harcèlement moral au travail est une donnée que l'on peut vérifier statistiquement, elle ne garantit pas une protection judiciaire réelle et efficace. La grande majorité des jugements rendus rejettent la demande des travailleurs, au motif qu'ils considèrent qu'il ne s'agit pas réellement de harcèlement au travail, mais plutôt de conflits typiques du travail, ou même d'une situation de forte tension. Etant donné que ce comportement peut être considéré comme portant atteinte aux droits fondamentaux (art. 15 de la Constitution espagnole - CE - : droit à l'intégrité personnelle) ou peut même devenir un délit, en Espagne, la position de la majorité des juges est de s'autolimiter, afin d'éviter que les conflits, ou les situations de simple exercice légitime du pouvoir de l'employeur, ne soient

1 Vid. EUROFOUND. «La violencia y el acoso laboral en Europa: alcance, repercusiones y políticas », 2015.

https://www.eurofound.europa.eu/es/observatories/eurwork/comparative-information/ violence-and-harassment-in-european-workplaces-extent-impacts-and-policies 
confondus avec du harcèlement. Ce qui explique qu'en général, il y ait davantage de résistances judiciaires que d'avancées dans ce domaine.

Cette étude a pour objectif de présenter un panorama complet, bien que synthétique, de l'état de la jurisprudence de la Cour suprême (TS ci-après) et celles des juridictions inférieures (Cours de justice supérieures autonomes - TTSSJ -) en Espagne concernant la protection contre le harcèlement au travail ${ }^{2}$. Dans ce cadre, trois aspects seront traités. Tout d'abord, nous délimiterons le concept judiciaire de harcèlement applicable dans un contexte juridico-social, en l'absence d'un concept légal spécifique, en sélectionnant les éléments constitutifs identifiés par le juge (I). Ensuite, nous développerons le fondement juridique privilégié par les tribunaux espagnols pour apporter une protection, à savoir le droit fondamental à l'intégrité (art. 15 CE) et les conséquences qui en découlent en pratique (II). Enfin, nous dresserons un inventaire des différentes qualifications juridiques que peut recevoir le harcèlement moral au travail afin de garantir une protection complète face à ce dernier : prévention, réparation et sanction (III).

\section{I - LE CONCEPT JURISPRUDENTIEL DE HARCÈLEMENT MORAL AU TRAVAIL : LA PRÉDOMINANCE DANS LA PRATIQUE JUDICIAIRE ESPAGNOLE DE LA NOTION EXTRA-JURIDIQUE OU CLINIQUE}

Faute d'un concept légal de harcèlement moral au travail (la législation espagnole définit uniquement de façon expresse le harcèlement discriminatoire, en s'alignant sur le Droit de I'Union), la doctrine judiciaire espagnole a adopté la notion fréquemment utilisée dans le domaine de la psychologie clinique qui met en avant l'asservissement de l'individu notion du psychologue suédois H. Leymann). La STSJ Catalogne 5207/2017, du 12 septembre le résume ainsi : "...le harcèlement moral ou mobbing peut être défini comme toute ... violence psychologique à laquelle une personne est systématiquement soumise dans le cadre professionnel, qui se manifeste en particulier par des comportements répétés, des mots ou des attitudes qui portent préjudice à la dignité ou à l'intégrité psychique du travailleur et qui ... dégradent ses conditions de travail ».

Ce concept jurisprudentiel s'écarte aussi bien de la définition du harcèlement discriminatoire (tout comportement ayant pour objet ou conséquence de créer un environnement intimidant, offensant ou dégradant pour une ou plusieurs personnes au travail, susceptible de porter préjudice à sa/leur dignité), que de l'aspect technicoscientifique aux fins de prévention des risques, selon l'affirmation de la Note technique préventive 854/2009 de l'organe technico-scientifique national principal en matière de santé et sécurité au travail (Institut national de sécurité, santé et bien-être au travail, INSSBT). Ce concept restrictif est également celui qui est retenu, pour le harcèlement moral à l'encontre des fonctionnaires, par la jurisprudence administrativo-contentieuse (STS, Chambre du contentieux, 16 février 2011, entre autres; également STSJ Castille-et-León, Chambre du contentieux, 356/2017, 24 février, etc.).

Ainsi, la jurisprudence espagnole reste éloignée du rôle qu'elle devrait jouer pour faire progresser les choses : elle n'est convenablement adaptée ni à l'évolution de la pensée scientifico-préventive, ni au traitement normatif du harcèlement discriminatoire. Cette

2 Une étude plus détaillée dans C. Molina Navarrette, La tutela judicial frente al acoso moral en el trabajo, Editorial Bomarzo, Albacete, 2018. 
dissociation conceptuelle n'est pas justifiée. En pratique, le recoupement des deux notions est donc fréquent : harcèlement moral et harcèlement discriminatoire. Par exemple, une situation de harcèlement moral est souvent associée à une autre de harcèlement sexuel (STC 250/2007, du 17 décembre), ou de discrimination fondée sur le sexe (STSJ Pays Basque 1608/2017, 18 juillet). II existe également des cas dans lesquels le harcèlement moral a pour cause une discrimination fondée sur la nationalité (ex. : STSJ Madrid, 1263/2017, du 20 décembre). Par conséquent, cette dissociation radicale manque de cohérence théorique et pratique.

En 17 ans, la jurisprudence espagnole n’a pas modifié les 4 éléments essentiels caractéristiques du harcèlement moral au travail :

a) Une composante chronologique : le caractère continu du comportement nuisible.

b) Une composante bilatérale : la dimension complexe du comportement, de sorte que l'environnement hostile porte préjudice aussi bien à la «facette professionnelle » du travailleur qu'à celle « relationnelle » de l'organisation.

c) Une composante subjective : l'intention de nuire.

d) Une composante de résultat : I'atteinte à la santé psychique du travailleur.

A notre sens, le grand problème de cette doctrine judiciaire majoritaire et erronée est de confondre les composantes structurelles du harcèlement - la création d'un cadre offensant ou intimidant et ses effets répétés dans le temps -, avec les composantes circonstancielles -l'intention de nuire et le préjudice qui en découle -. Sans les premières, la notion juridique de harcèlement moral n'existerait pas, alors que ces dernières ne servent qu'à aggraver les conséquences juridiques (cf. STSJ Castille-et-León/Burgos, 218/2014, du 8 avril, courant minoritaire). Cette notion plus objective de harcèlement moral, qui relègue à un deuxième plan l'intention de harceler, est celle adoptée par l'arrêt de la Cour constitutionnelle - STC - TC 250/2007, du 17 décembre, ainsi que par des décisions judiciaires qui font primer la composante de l'habitude et l'intensité du comportement. On constate ainsi le harcèlement au travail : « (...). Quand cette faculté d'organiser le travail de ses salariés ... est exercée d'une façon susceptible de provoquer une maladie ...de l'anxiété [préjudice physique et moral] chez...une de ses salariées... » (TS, Chambre sociale, du 11 octobre 2017, RCUD 3627/14).

\section{II - LE PRINCIPAL MOTIF JURIDIQUE DE PROTECTION JUDICIAIRE : LA PROTECTION POUR VIOLATION DU DROIT FONDAMENTAL À L'INTÉGRITÉ PERSONNELLE}

Bien que la pratique judiciaire espagnole continue à se montrer réticente à qualifier juridiquement des situations de harcèlement au travail, en les considérant comme des comportements exceptionnellement anormaux ou d'une conflictualité très singulière, quand elle constate des cas authentiques de harcèlement au travail elle leur apporte une protection supplémentaire. Le motif de cette protection renforcée réside dans l'identification d'une atteinte aux droits fondamentaux, plus particulièrement le droit à I'intégrité psychophysique et morale (art. 15 CE ; STSJ Galice, 20 juillet 2017, Rec. 542/17)). Plus encore, la jurisprudence et la doctrine judiciaire espagnoles reconnaissent que le harcèlement moral est une forme de comportement constituant une atteinte à plusieurs droit fondamentaux constitutionnellement reconnus : intégrité personnelle (art. $15 \mathrm{CE}$ ), liberté de communication (art. $20 \mathrm{CE}$ ), discrimination (art. $14 \mathrm{CE}$ ), honneur (art. $18 \mathrm{CE}$ ) et même la protection judiciaire (art. $24 \mathrm{CE}$ ), en raison de la fréquence des harcèlements au 
travail exercés en représailles par l'entreprise (ex. STSJ Andalousie/Séville, 3581/2016, 20 décembre).

Dans tous les cas, la doctrine constitutionnelle (STC 160/2007, du 2 juillet) fonde la protection face au harcèlement moral sur le droit à l'intégrité (art. 15 CE) (pour le cas particulier du harcèlement discriminatoire, le fondement de la protection supplémentaire réside dans l'art. $14 \mathrm{CE}$ ).

La jurisprudence espagnole se fonde sur l'inconstitutionnalité non seulement pour accorder une protection spéciale en cas de harcèlement, mais également pour établir la différence même entre le harcèlement et un autre type de pratiques ou de comportements qui soit n'ont pas besoin de protection (conflits du travail impliquant une forte tension) soit ne doivent recevoir qu'une protection juridique de droit ordinaire. En résumé, les comportements conflictuels au travail, qualifiables de harcèlement moral, se différencient d'autres pratiques conflictuelles créatrices de risques pour la santé au travail, du fait que ces comportements portent atteinte aux droits fondamentaux. Les attitudes qui ne reçoivent pas la qualification de harcèlement ne permettront que la mise en jeu du « droit ordinaire du travail » (SSTTSSJ Galice 11 octobre 2017 ; Catalogne 5207/2017, du 12 septembre ; etc.). En d'autres termes, dans l'apport jurisprudentiel espagnol, il faut différencier le harcèlement moral d'un vaste ensemble de conflits normaux ou anormaux qui seront résolus sur le fondement du droit commun (la STSJ Andalousie/Séville, 1623/2017, 31 mai, les qualifie tous de « dégradations normalisées de gestion du personnel »). A savoir :

1. Les conflits liés aux conditions de travail (lieu, temps, modalités de la prestation, etc.), qui constituent une " dégradation normalisée de la relation de travail " (SSTTSJ Madrid 485/2017, 24 juillet, Andalousie, 3581/2016, 20 décembre). Le conflit peut être un indice du harcèlement, jamais déterminant (STSJ Catalogne 2540/17, 19 avril).

2. Les situations de pression professionnelle prolongées génératrices de stress (SSTTSSJ Galice 1027/2010, du 10 mars, entre autres).

3. Les modes d'exercice illégaux - abusif ou arbitraire - du pouvoir de l'employeur. II s'agit de toutes les formes d'exercice inadéquat, déplacé ou anormal des facultés de l'entreprise orientées par "l'intérêt économique de l'entreprise », mais sans la volonté ou l'intention de perturber la stabilité émotionnelle du travailleur ni de créer un environnement global dégradant (STSJ Galice, 11 octobre 2017).

4. Les formes d'exercice tyrannique et humiliant du pouvoir de direction de l'employeur (ou d'un autre pouvoir dans l'entreprise), causant des préjudices psychiques aux travailleurs pris isolement, bien qu'exercé contre la collectivité des travailleurs sous son autorité (SSTSJ Madrid 485/2017, 24 juillet, op. cit. et 716/2017, 5 juillet). Cependant, si dans un contexte conflictuel général, le juge identifie des «faits humiliants singuliers », il est possible de les qualifier « d'abus d'autorité constitutifs de harcèlement »(STSJ PV 1660/2017, 5 septembre).

Malgré la surprenante constance de cette jurisprudence, le droit prétorien espagnol le plus récent laisse augurer de quelques évolutions. Nous en présenterons trois :

1. Le plus important pour que la qualification juridique d'atteinte aux droitsfondamentaux soit retenue n'est pas le nom donné au comportement mais sa nature. L'employeur est responsable juridiquement "que le comportement soit qualifié de harcèlement moral « stricto sensu » ou non, l'obligation ... de réparer le préjudice causé ... s'impose ... »(STSJ PV 1608/2017, 18 juillet; STSJ Madrid 581/2014, op. cit.)

2. Toute situation de conflit du travail prolongé susceptible de causer un préjudice psychosocial, sans l'intervention préventive due par l'employeur, constitue un manquement 
contractuel non seulement au droit commun - devoir de protection efficace du droit à la santé -, mais également au droit constitutionnel - devoir de protection de l'intégrité personnelle - (STSJ PV 1660/2017, 5 septembre). Ce courant, aujourd'hui minoritaire, serait équivalant à la doctrine jurisprudentielle française résultant de l'arrêt de la Chambre sociale de la Cour de cassation du 6 décembre 2017 n¹6-10891.

3. On note la prise en considération judiciaire d'une nouvelle forme de harcèlement numérique au travail - liée à la discrimination sexuelle - (SSTTSSJ Castille-La Manche, 443/2016, du 8 avril et Pays Basque 1646/2017, 18 juillet (un salarié harcèle une collègue en utilisant le numéro de téléphone de l'entreprise et WhatsApp).

\section{III - LA TRIPLE VOIE DE LA PROTECTION JUDICIAIRE CONTRE LE HARCÈLEMENT MORAL AU TRAVAIL DANS LA PRATIQUE ESPAGNOLE : PRÉVENTION, RÉPARATION ET SANCTION}

Idéalement, tout système juridique comprend trois types de protections efficaces contre le harcèlement moral au travail. A savoir :

1. Les techniques de protection préventive. En effet le harcèlement au travail est un risque professionnel de type psychosocial, que l'employeur doit prévenir efficacement sur la base du système de gestion de la prévention des risques professionnels (art. 14 de la Loi de prévention des risques professionnels - LPRL -)

2. Les techniques de protection réparatrice face aux préjudices causés par les comportements de harcèlement au travail qui n'ont pas fait l'objet de prévention. La protection face aux dommages causés au travailleur qui découlent du harcèlement se présente, elle aussi, à deux niveaux : la qualification comme maladie du travail, avec la prestation de Sécurité Sociale qui en découle afin d'indemniser les arrêts de travail occasionnés, d'une part, et la responsabilité civile ou professionnelle de l'employeur pour lesdits préjudices découlant d'un manquement grave et coupable aux obligations en matière de comportement et de protection relevant de la responsabilité de l'employeur, d'autre part.

3. Les voies de protection punitive. Lorsque le harcèlement au travail constitue une infraction punissable, qu'elle soit administrative - le harcèlement en tant qu'infraction grave de l'employeur -, disciplinaire - le harcèlement d'un collègue de travail -, ou pénale - le harcèlement moral en tant que délit de traitement humiliant ex art. $173 \mathrm{CP}$.

Dans la pratique judiciaire espagnole, tous ces moyens de protection sont appliqués, avec différents niveaux d'intensité et de succès pour les travailleurs victimes. Le tableau récapitulatif de cette riche expérience judiciaire de protection, bien que présentant des résultats très variables, serait le suivant:

\section{A - LE HARCÈLEMENT COMME RISQUE PROFESSIONNEL SPÉCIFIQUE DANS LE DROIT JUDICIAIRE ESPAGNOL}

Dans un système juridique tel que le système espagnol, qui ne dispose pas de règlementation spécifique concernant les risques psychosociaux, il y a d'abord eu des doutes judiciaires sur sa qualification comme risque professionnel (STS, Chambre du social, 15 décembre 2018). Cependant, bien que la législation continue de l'ignorer, la jurisprudence reconnaît désormais que le harcèlement est un risque psychosocial que l'entreprise doit gérer (ex. : STSJ Catalogne 7055/2017, 20 novembre), et qui doit par 
conséquent être inclus dans l'évaluation des risques (STS, Chambre du social, 101/2016, du 16 février). Mieux encore, si le risque de harcèlement moral n'est pas inclus dans la politique de prévention de l'entreprise, même en l'absence de préjudice, l'employeur commet une infraction administrative grave, punie par des amendes, et en cas de préjudice causé à la victime, l'entreprise devra payer, en plus de l'indemnisation des préjudices, le "supplément de prestations de la Sécurité Sociale " (art. 164 de la Loi générale de la Sécurité Sociale - LGSS -), qui agira à titre de « dommages punitifs » et dissuasifs (l'employeur doit payer, directement à sa charge, entre 30 et $50 \%$ de la prestation correspondante aux arrêts maladie découlant du harcèlement: STS, Chambre du social, 4 mars 2014, RCUD 788/2013).

En Espagne, il n'existe pas de règlementation juridique imposant à l'employeur de mettre en place une politique d'intervention préventive contre le harcèlement moral au travail - s'il est lié au harcèlement sexuel ou sexiste -. Toutefois, lorsqu'elle existe, la doctrine judiciaire tend à la considérer comme une exonération de responsabilité en cas de harcèlement, ou du moins comme une mesure qui réduirait sa responsabilité (STSJ Madrid 877/2017, 16 octobre ; STSJ Murcie 446/2017, du 26 avril), dans la mesure où elle a été exercée de façon correcte (STSJ Galice 9 novembre 2017).

\section{B - LES PRÉJUDICES CAUSÉS PAR LE HARCÈLEMENT AU TRAVAIL COMME MALADIE DU TRAVAIL (ART. 156.2 E) LGSS), ET NON COMME MALADIE PROFESSIONNELLE (ART. 157 LGSS)}

Le Droit espagnol, qui est basé sur un système de listes, ne reconnaît pas non plus la qualification de maladie professionnelle aux préjudices ou pathologies de harcèlement moral au travail, car ils ne sont pas inclus dans la liste spécifique à cette fin (art. 157 LGSS), raison pour laquelle le bénéfice de la présomption légale n'est pas reconnu. Cependant, la jurisprudence leur a reconnu la nature de "maladie du travail » - à condition que le travailleur victime prouve lors d'un procès le lien de cause à effet avec le harcèlement en leur apportant une protection renforcée comme s'il s'agissait d'un accident du travail (art. 156 LGSS). Ici la jurisprudence a également accompli, depuis 2011 jusqu'à ce jour, un travail évolutif qui compense les carences légales pour s'adapter à la réalité (ex. : STSJ Castille-et-León, 15 septembre 2017, Rec. 703/2017, pour les travailleurs du secteur privé ; STS, Chambre du contentieux, 15 mars 2011, pour les fonctionnaires).

\section{C - LE HARCÈLEMENT COMME MANQUEMENT CONTRACTUEL GRAVE ET L'OBLIGATION À CHARGE DE L'EMPLOYEUR DE DÉDOMMAGER LES PRÉJUDICES DE FAÇON INTÉGRALE ET DISSUASIVE (ART. 183 LRJS)}

En plus de ce système de responsabilité objective pour les préjudices découlant du harcèlement moral au travail (ou de tout type de harcèlement et/ou violence au travail, également de la part de tiers), qui nécessite la seule constatation du préjudice, indépendamment du fait qu'il y ait culpabilité de l'employeur ou pas, le droit jurisprudentiel espagnol applique un système de responsabilité (civile) pour faute parce que l'employeur est l'auteur du harcèlement ou parce qu'il n'a pas éradiqué le harcèlement perpétré par des collègues ou des tiers envers le travailleur sous sa direction, et par conséquent sous sa protection. L'art. 183 de la Loi règlementant la juridiction sociale - LRJS - prévoit désormais, que tout comportement violant les droits fondamentaux (non seulement le harcèlement mais également tout préjudice, patrimonial ou moral) entrainera l'obligation d'indemniser de façon intégrale le préjudice-. En outre, l'indemnisation remplit un rôle dissuasif, à 
savoir qu'elle contribue à ce que de tels cas ne se reproduisent plus à l'avenir, ni dans l'entreprise condamnée, ni dans aucun autre. La jurisprudence (SSTS, Chambre du social, 20 septembre 2011, 8 mars 2017, etc.) et les doctrines judiciaires autonomes (ex. : STSJ Andalousie/Séville, 1662/2017, 1 er juin, STSJ Catalogne 2686/2017, 24 avril, etc.) n'hésitent pas à en faire application.

Cependant, en pratique, les tribunaux espagnols fixent des indemnisations réduites (autour de 12000 euros en moyenne, bien que les montants soient très variables - allant de 6000 jusqu'à 50000 euros), et l'effet dissuasif-préventif, à ne pas confondre avec les «punitive damages ${ }^{3}$, est à peine pris en compte. Par conséquent, à cet égard, la jurisprudence ne remplit pas un rôle adéquat de garantie ni d'efficacité de la protection.

\section{D - LE HARCÈLEMENT MORAL COMME INFRACTION ADMINISTRATIVE GRAVE ET/OU DÉLIT DE TRAITEMENT HUMILIANT}

La critique que de nombreux travailleurs expriment précisément à l'égard de la pratique judiciaire sociale - et contentieuse, pour les fonctionnaires - contre le harcèlement au travail, en raison de son inefficacité, a conduit plusieurs d'entre eux à porter plainte par voie administrative (infractions et sanctions publiques sur proposition de l'inspection du travail - ITSS - et application par les autorités du travail), et à utiliser aussi la voie pénale. Le Droit administratif social répressif n'envisage pas non plus de façon spécifique le harcèlement moral, à part le harcèlement discriminatoire ; mais il inclut des infractions génériques (d'une grande gravité) qui peuvent trouver application : I'atteinte à la dignité des travailleurs (art. 8 du texte refondu de la Loi sur les infractions et sanctions d'ordre social - TRLISOS -). A cet égard, il est fréquent que I'ITSS sanctionne un bon nombre de pratiques entrepreneuriales qualifiées de harcèlement (Critère technique 69/2009), mais qui sont régulièrement validées par les Tribunaux.

Pour le harcèlement moral au travail de plus forte intensité, le Code pénal espagnol (CP), envisage depuis 2010 un délit spécifique, art. 173.1 CP (pour le harcèlement sexuel, il prévoit un autre art. $184 \mathrm{CP}$ ), et, depuis 2015, un délit de harcèlement par internet ou «stalking » (art. 172, ter 2 CP ; STS, Chambre du pénal, 324/2017, du 12 juillet). La jurisprudence pénale a procédé à des condamnations pour harcèlement au travail, sur le fondement du délit de traitement humiliant (art. 173 CP), aussi bien avant l'existence d'une catégorie pénale spécifique (ex. : STS, Chambre du pénal, 945/2010, du 28 octobre) qu'après (ex. : STS, Chambre du pénal, 325/2017, 19 septembre). II apparaît donc que les possibilités de protection contre le harcèlement moral ne varient pas beaucoup, du moins en Espagne, peu important qu'il existe ou non une législation spécifique.

Dans tous lescas, force est de constater le nombre extrêmement faible de condamnations pénales pour harcèlement au travail, la grande majorité des plaintes étant classées sans suite. Pour un exemple récent de condamnation, il faut se référer au Jugement de l'audience provinciale de Cáceres - Estrémadure -, 18/2018, du 9 janvier, qui a condamné un maire pour le double délit d'humiliations et d'atteinte à l'intégrité psychique pour harcèlement (il avait dénigré et isolé une fonctionnaire et ne lui avait fourni aucun équipement de travail pour effectuer ses missions). Il fut condamné à une peine de prison d'un an et demi - la peine maximale prévue est de deux ans - et une indemnisation de 4000 euros. Mais, nous insistons, la plupart du temps, cette voie s'achève par une déception du salarié.

3 Selon la STJUE du 17 décembre 2015, affaire Arjona Camacho, C-407/14. 


\section{Conclusion : un tableau de lumières (rares) et d'ombres (multiples)}

Ce passage en revue de la pratique espagnole en matière de harcèlement au travail nous a permis de constater à quel point le corpus de la doctrine jurisprudentielle existant est riche. Malgré l'absence persistante d'une législation spécifique, sauf dans le domaine pénal - et une certaine référence confuse dans les lois sur l'emploi public, depuis 2007, à peine prises en compte par les juges et les tribunaux -, le droit prétorien anti-harcèlement offre un très vaste catalogue de formes de protections aux travailleurs victimes de harcèlement moral sur les lieux de travail. Le résultat de cet activisme judiciaire espagnol est la conformation d'un statut de protection renforcée, aussi pertinent et protecteur que celui que pourraient offrir les systèmes règlementaires européens qui, eux, comportent des législations spécifiques.

Le problème est qu'en pratique, ce statut théorique de protection renforcée, conçu à partir de l'adaptation jurisprudentielle des techniques communes de protection des droits fondamentaux, ne s'applique pas, car il implique des conditions trop rigoureuses pour qualifier le comportement de harcèlement. Pour la jurisprudence, il ne peut y avoir harcèlement que dans des situations de conflit intense et dans un cadre gravement dégradé, avec l'intention spécifique de porter préjudice au travailleur. En conséquence, face à une telle exigence, qui ne serait pas justifiée sur le plan règlementaire ni conceptuel, la grande majorité des demandes est rejetée. Par conséquent, si la délimitation jurisprudentielle espagnole est très différente des autres délimitations européennes (comme, par exemple, la jurisprudence italienne, qui exige un dol ou une intention spécifique de porter préjudice à la personne: Cour de cassation italienne, du 10 novembre 2017, $n^{\circ} 26685$ ) il est nécessaire d'exiger une modification de cette conception jurisprudentielle trop rigide, afin de mettre en œuvre efficacement l'obligation de protection contre le risque de harcèlement moral, comme l'exige le droit fondamental à l'intégrité personnelle concernant la santé des personnes (ex art. 15 CE relatif à l'art. 43 CE) 